\title{
OPTIMAL CHOICE OF SUPERVISED TECHNIQUES FOR MR IMAGE CLASSIFICATION
}

Balasubramanian Aruna Devi

Electronics and Communications engg, Kalasalingam Academy of research and Education, Krishnankoil, (India). E-mail: b.arunadevi@klu.ac.in ORCID: https://orcid.org/0000-0002-0981-804X

\section{Murugan Pallikonda Rajasekaran}

Professor. Electronics and Communications engg, Kalasalingam Academy of research and Education

Krishnankoil, (India). E-mail: m.p.raja@klu.ac.in ORCID: https://orcid.org/0000-0001-6942-4512

\section{Citación sugerida:}

Devi, B. A., y Rajasekaran, M. P. (2020). Optimal choice of supervised techniques for MR image classification. 3C Tecnología. Glosas de innovación aplicadas a la pyme. Edición Especial, Marzo 2020, 313-327. http://doi.org/10.17993/3ctecno.2020.specialissue4.313-327

\section{Suggested citation:}

Devi, B. A., \& Rajasekaran, M. P. (2020). Optimal choice of supervised techniques for MR image classification. 3C Tecnología. Glosas de innovación aplicadas a la pyme. Edición Especial, Marzo 2020, 313-327. http://doi.org/10.17993/3ctecno.2020.specialissue4.313-327 


\section{ABSTRACT}

Magnetic Resonance Imaging (MRI) is a modern, robust method that uses in the detection of various medical problems. In this research work, a trial is used to attempt for the detection of tumour in pancreas MR images. An automated classifier is used for detection of tumour in MR images and avoids the drawbacks of MRI. This automated classifiers can detect automatically, either the MR image is affected or not affected. Features are extracted from MR images using second order statistics approach and are classified by two techniques Support Vector Machine (SVM) and Extreme Learning Machine (ELM). SVM approach has high classification accuracy $(96 \%)$ which is higher than ELM, while ELM performs faster compared to SVM.

\section{KEYWORDS}

SVM, ELM, GLCM feature extraction, Image classification. 


\section{INTRODUCTION}

In medicine field, medical Image analysis and processing has enormous applications. It has emerged as one of the superior tools to diagnose as well as detect many disorders. It permits both radiologists and doctors to make exact detection, by analyzing and visualizing the medical image. Computer-Aided Diagnosis (CAD) is an approach that is achieving attention in modern life. It can comfort doctors accurately read images and diagnosis possible decisions to avoid incorrect understanding of lesions. It is necessary to mark that CAD systems can only present a second opinion and can by no means follow physicians or radiologists. There are many imaging modalities for the humans of tissue analysis, such as Magnetic Resonance Imaging (MRI), mammogram function, Computed Tomography (CT) and so on. The main target of this research work is on MRI images. MRI (Armstrong, Cohen, Weinbrg, \& Gilbert, 2004) is a medical imaging method that generates images of the inner part of human body. It is an on-radio active, non-aggressive, pain-free method for visualizing detailed data regarding the normal or tumors without any human involvement. The target of this research work is to grant an automatic detection tool that will guide physicians or radiologists in detecting lesions by diagnosing them from normal tissue. The first step is to extract the features in MRI Images by second order statistics. These extract important image features from the MR Images are used to classify the image is affected or not affected. This will help the physicians or radiologists in the analysis of diagnosing tumor in MR images. In this research work, we have analyzed two classifiers such as SVM (Vapnik, 1995) \& ELM (Huang, Zhu, \& Siew, 2004).

\section{SVM}

SVM is a classification approach for high-dimensional data which is presented by Vapnik (1995) to resolve the discrimination disputes of two issues. SVM has been broadly used in the fields of medical image processing, image retrieval, text analysis, and so on. SVM is based on the working principle that the data in the input space can be linear dividable in a higher dimensional feature space after a certain mapping. 


\section{ELM}

ELM (Huang et al., 2004) is a newly advanced machine learning approach, extensively applied in image processing, computer Vision, biomedical applications, system modeling and regression.

The first stage describes with the feature extraction applying GLCM in MRI pancreas images. In the second stage, they are discriminated by the classification methods such as SVM \& ELM. The output displayed that SVM classification method has the superior classification accuracy compared to ELM.

\section{LITERATURE SURVEY}

Lu et al. (2017) presented DWT feature extraction and classified by bat algorithm based extreme learning machine with a classification accuracy of 93.33\%. Nazir, Wahid, and Khan (2015) classified brain MRI using various moment features extraction and artificial neural networks with an accuracy of 94.24\%. Nandpuru, Salankar, and Bora (2014) classified brain tumor applying Texture features, symmetrical and gray features extraction, principal component analysis (PCA) feature selection and support vector machine (SVM) classification. The classification accuracy was 84\%. Ibrahim, Osman, and Mohamed (2018) classified MR brain images using wavelet-based features extraction, features selection by PGA, and classified by artificial neural networks (ANN) with a classification accuracy of 96.33\%. Othman, Abdullah, and Kamal (2011) discriminated normal and abnormal MRIs using DWT feature extraction, principal component analysis (PCA) feature selection and SVM classification by 65\% classification accuracy. Kavitha and Thyagharajan (2012) have presented histogram, textural features and classified by SVM with an accuracy of 90\%. Diz, Marreiros and Freitas (2015) have described GLCM and Grey-Level Run Length Matrix (GLRLM) feature extraction for mammogram image classification and achieved 76\% accuracy. Dheeba and Selvi (2011) have presented Laws texture features to discriminate images into Benign and Malignant (MIAS-Mammographic Image Analysis Society database) and gained 86.10\% accuracy. Shah, Surve and Turkar (2015) classified pancreatic tumor of CT images using Minimum distance classifier. The classification accuracy was 61.59\%. Yao, Chen and Chow (2009) described wavelet transform features extraction method and classified by SVM with an accuracy of $83 \%$. Aruna Devi and Pallikonda 
Rajasekaran (2018) described GLCM features extraction and differentiated normal and abnormal MR images by applying ANN and SVM methods. ANN found that it achieves 96\% classification accuracy. Aruna Devi, Pallikonda Rajasekaran and Thiyagarajan (2019) proposed tumor discrimination by GLCM feature extraction, JAFER feature selection and comparison among 5 types of classification modes, ANN BP gains 98\% classification accuracy. Based on the survey, the discrimination capability of classifiers is very less and computational time is also high. Our proposed technique increases the discrimination capability as high $(98 \%)$ that is superior to previous research analysis. In this research work, we are going to discriminate the $160 \mathrm{MR}$ images by affected or not affected using second order statistics feature extraction and discriminated by two modes such as ANN and SVM. Sensitivity, specificity and classification accuracy is measured and compared among the two modes.

\section{MATERIALS AND METHODS}

Input data set:

The dataset used for predicting the performance of the proposed model in this research work is based on the MR pancreas medical images that are gathered from the health care centres. The numbers of medical pancreas images totally 160 of which 100 are normal and 60 are abnormal images. Figure 1 shows the normal pancreas images and Figure 2 shows the abnormal pancreas images. Figure 3 displays the flowchart.

\section{FEATURES EXTRACTION}

Features extraction by second order statistics:

The procedure of transferring the input image into a set of features is known as feature extraction. Features normally consist of data relevant to colour, shape, texture or context. First order statistics provides gray level pixels occur in an image. First order measures are mean, variance, skewness and kurtosis. Second order statistics provides inter relationship between pixel and its neighbors. They provide detailed information about the pixel and its neighbors with an angle of 0, 45, 90 and 135 degrees at a distance d. Second order measures are entropy, energy, contrast, homogeneity, sum of variance, cluster prominence, 
sum of entropy, cluster shade, information measure of correlation. Second order statistics features are tabulated in Table 1. In this work, these features are measured and classified by two classifiers namely SVM and ELM.

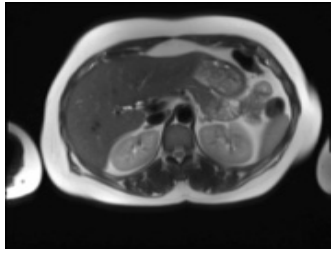

a

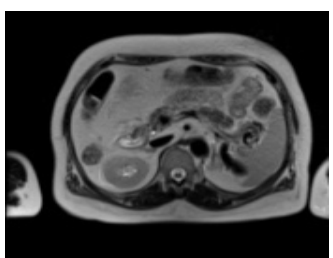

d

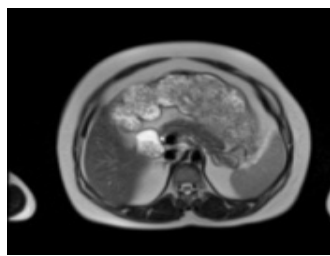

g

Figure 1. Normal pancreas MR images.

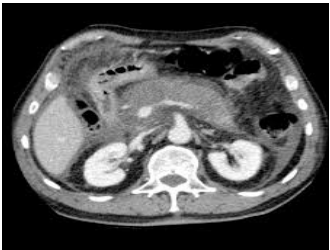

a

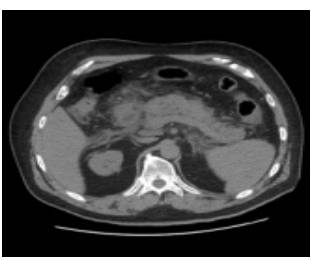

d

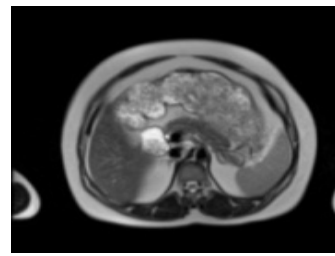

b

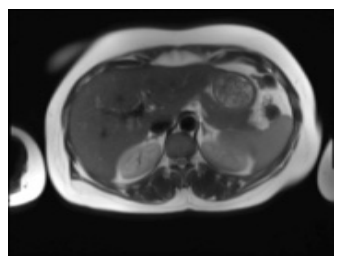

e

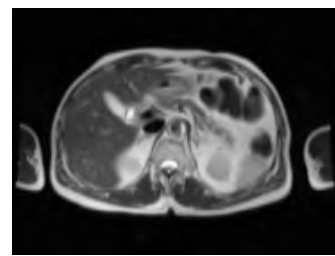

h

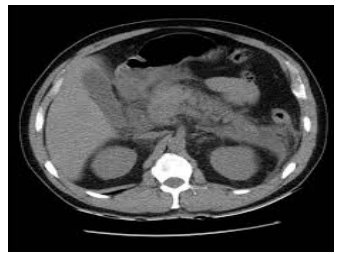

b

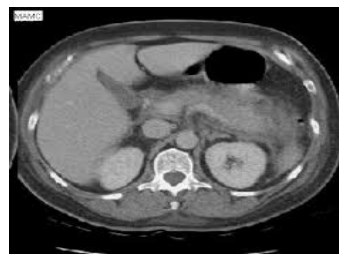

d

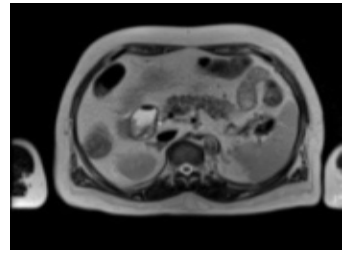

C

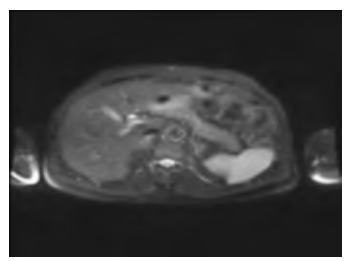

f

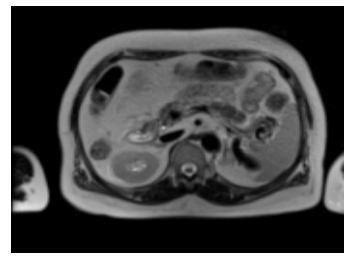

i

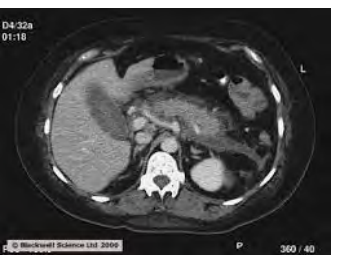

c

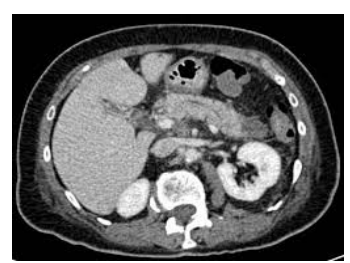

$\mathrm{f}$ 


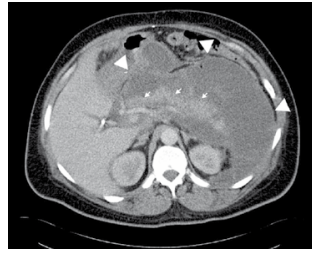

g

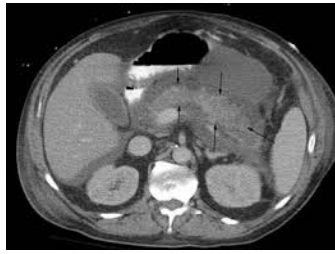

h

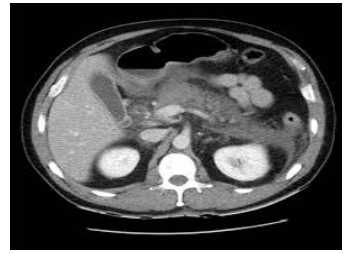

i

Figure 2. Abnormal pancreas MR images.

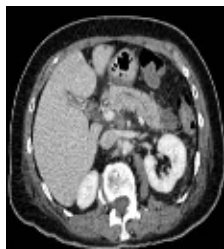

Features extraction using second order statistics

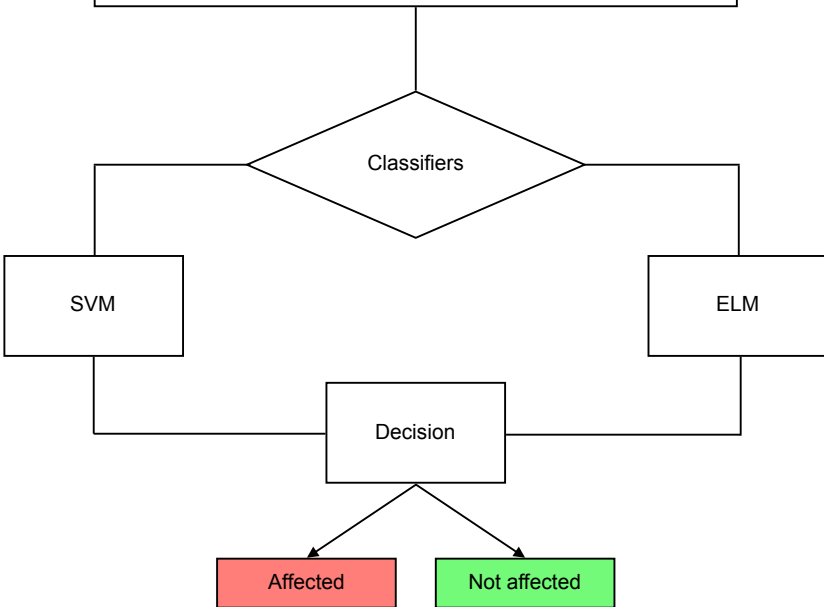

Figure 3. Flow chart.

Table 1. GLCM features.

$$
\begin{aligned}
& \text { GLCM features: } \\
& \text { Contrast: } \quad \sum_{i} \sum_{j}(i-j)^{2} g_{i j} \\
& \sum_{i} \sum_{j}(i-j)^{2} g_{i j}(1) \sum_{i} \sum_{j}(i-j)^{2} g_{i j} \sum_{i} \sum_{j}(i-j)^{2} g_{i j} \sum_{i} \sum_{j}(i-j)^{2} g_{i j} \\
& \text { Energy: } \quad \sum_{i} \sum_{j} g_{i j} \sum_{i} \sum_{j} g_{i j^{2}} \\
& \text { Entropy: } \quad \sum_{i} \sum_{j} g_{i j} g_{i j} \sum_{i} \sum_{j} g_{i j} g_{i j} \\
& \sum_{i} \sum_{j}(i-j)^{2} g_{i j} \sum_{i} \sum_{j}(i-j)^{2} g_{i j} \sum_{i} \sum_{j} g_{i j} g_{i j} \\
& \text { Homogeneity: } \left.\left.\quad \sum_{i} \sum_{j} \frac{(1}{1+(i-j)^{2}}\right) g_{i j}: \sum_{i} \sum_{j} \frac{(1}{1+(i-j)^{2}}\right) g_{i j} \\
& \left.\sum_{i} \sum_{j} \frac{(1}{1+(i-j)^{2}}\right) g_{i j}
\end{aligned}
$$




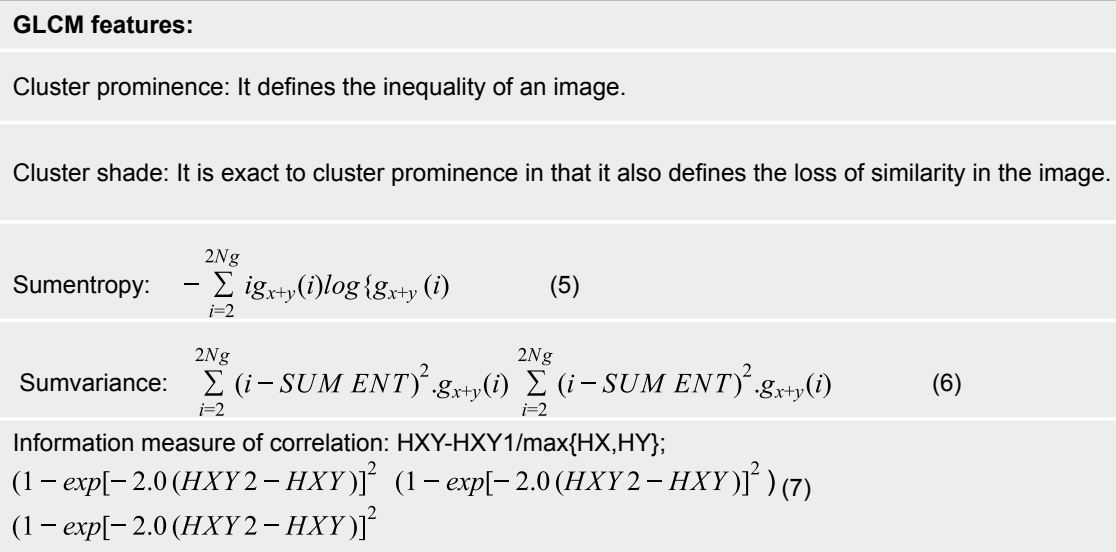

\section{CLASSIFICATION}

Classification is the procedure of classifying a known input by a good classifier. The main target of proper classification is to provide a label to each MR image based on second order statistics features.

\section{SVM}

SVM is used for mapping complex feature space into linear feature space. It works on the base of fitting a boundary to a field of points that are belongs to one class with one another. Once boundary is fixed, on the learned samples, for any unknown points that are test sample need to be classified, and the accuracy will be predicted. Once boundary is fixed, maximum training points are redundant. All it demands a group of points that can identify and fits the boundary. The group of points are known as support vectors and the boundary is called as hyperplane.

\section{ELM}

Extreme Learning Machine (ELM) is a single hidden-layer feed-forward neural network (SLFN). The significance of the SLFN should be convenient for information such as weight, threshold value, and activation function so that superior training can be achieved. In gradient-based learning, all of these quality measures are changed iteratively for the significant value. Therefore, due to the possibility of being attached to the slow and local minimum, the performance can generate low outputs. On the basis of the gradient in the ELM training process, the output weights are analytically calculated where the input 
weights are chosen randomly. In this process, the success rate raises because the resolution time and the errors can actively shorten the possibility of being fitted to a local minimum.

\section{TRAINING AND TESTING}

Feature dataset is classified by the classification such as SVM \& ELM techniques. Out of 165 pancreas MR images, $70 \%$ Images were used for learning and $30 \%$ of images were used for testing.

There are more classification techniques, where training dataset are favoured by random sampling with restoration for MR pancreas image classification. Basically, choosing the best feature election approach with its performance parameters, it was required for process of classification approaches. In continuity, the classification outcomes with parameters for each discrimination approach are followed. Performance parameters were employed for each discrimination approach and the best one was choose for tumour identification.

Support vector machine is relevant for high dimension small-sample learning and nonlinear problems. SVM mainly focus at binary classification. SVM provides strong generalization ability and structural risk minimization. It separates the two classes using hyper plane. The second order statistics features are used as input of the classifier and the corresponding known label (that is affected or not affected) is the output of the classifier. The hyper plane separates the class as affected or not affected. It helps to train the classifier as input, output mappings functions. It provides the superior minimum distance to the learning data. The SVM has many kernel functions. The predominant kernel function is RBF that denotes radial basis function. Here RBF kernel is used. After learning the classifier, the test set was applied to test the significance of the classifier and its capability to accurately discriminate the MR images as either affected or not affected. To check out our SVM classifier, a confusion matrix was generated as shown in Figure 4 and the classification accuracy is $96 \%$ which is tabulated in Table 2.

\section{ELM}

Conventional single hidden-layer feed forward neural networks (SLFNs), such as the back propagation (BP) method, have been applied for research in many applications. The weight which assigns the hidden nodes is applied randomly and weights are not altered forever. 
The weights that correlate hidden nodes with outputs are trained in one step. ELM yields cooperative training technique. Even though ELM confirms to be terribly quick and in a great way in observation, it has some drawbacks. The election of hidden neurons is placed on trial and error technique that can provide into inadequate results. ELM provides 89\% classification accuracy.

Next, the performance evaluation approaches applied to test the two methods (SVM \& ELM). We analyse the execution of the methods in terms of sensitivity, specificity and accuracy.

\section{Performance opinion parameters:}

$$
\begin{gathered}
\text { Sensitivity }=T P /(T P+F N) 100 \% \\
\text { Specificity }=T N /(T N+F P) 100 \% \\
\text { Accuracy }=(T P+T N) /(T P+T N+F P+F N) 100 \%
\end{gathered}
$$

Where:

TN $($ True Negative $)=$ perfectly discriminated negative cases, $\mathrm{TP}($ True Positives $)=$ perfectly discriminated positive cases, FN (False Negative) = imperfectly discriminated positive cases FP (False Positives $)=$ imperfectly discriminated negative cases.

Specificity measures how perform the system can predict the negatives. Sensitivity is the rate of perfectly discriminated positives, describes best performance of the approach in predicting positives. Accuracy confirms the whole correctness of the classifier in predicting both positive and negative cases in terms of tumor.

\section{RESULTS}

SVM and ELM are learned by second order statistics features and classify the pancreas MR image as affected or not affected (normal or abnormal). SVM method provides good accuracy than ELM. The accuracy of SVM method is $96 \%$, specificity and sensitivity are $95 \%$ and $97 \%$ respectively. The classification accuracy of ELM is $89 \%$, specificity and sensitivity are 95\%and 92\% respectively. Table 2 represents classification accuracy, sensitivity and specificity for two methods. Figure 4 represents SVM confusion matrix. Figure 5 represents ELM ROC curve is graphed as a plot of true-positive rate on the y-axis and false-positive rate on the $\mathrm{x}$-axis. 


\section{CONCLUSION}

Among the two methods, SVM is the superior method which gives $96 \%$ classification accuracy. It proves that our proposed SVM technique gives high accuracy of $96 \%$, which is compared to other previous techniques. In this work, using second order stastics features are extracted and classified by SVM and ELM. Results are compared and proved SVM provides high classification accuracy of $96 \%$. In future, best features are selected using feature reduction methods (forward selection, backward elimination) and are classified by SVM and other classifiers to realize which classifier is best in practice.

\section{FUTURE WORK}

In this work, using second order statistics features are extracted and classified by SVM and ELM. Results are compared and proved SVM provides high classification accuracy of $96 \%$. In future, best features are selected using feature reduction methods (forward selection, backward elimination) and are classified by SVM and other classifiers to realize which classifier is best in practice.

Table 2. Comparative analysis of SVM and ELM techniques.

\begin{tabular}{|c|c|c|c|c|}
\hline $\begin{array}{c}\text { Classification } \\
\text { Techniques }\end{array}$ & $\begin{array}{c}\text { Classification } \\
\text { accuracy }\end{array}$ & Sensitivity & Specificity & Time \\
\hline SVM & 96.67 & 97.3 & 95.65 & 1.020 s \\
\hline ELM & 89.05 & 92.3 & 95.24 & 0.320 s \\
\hline
\end{tabular}

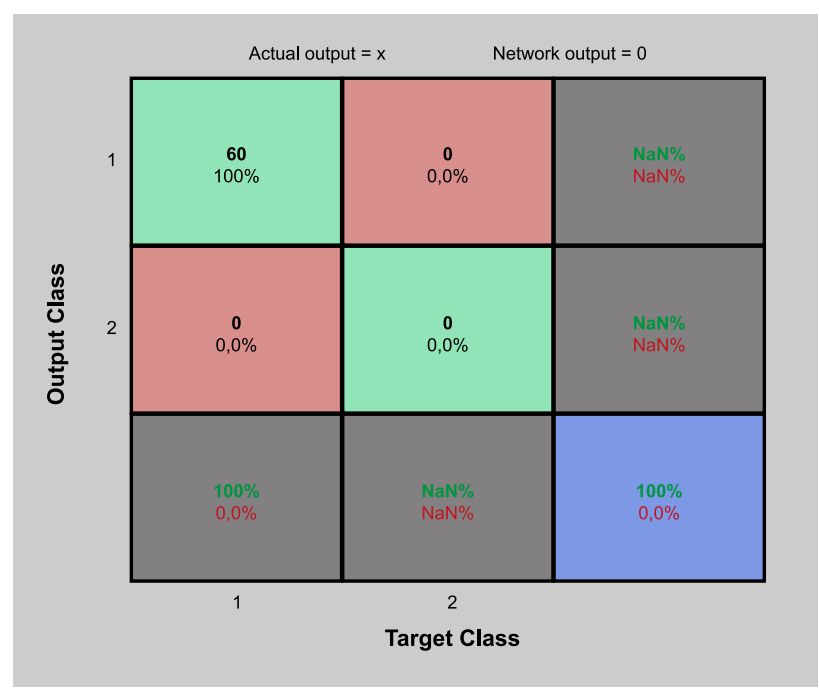

Figure 4. SVM confusion matrix. 


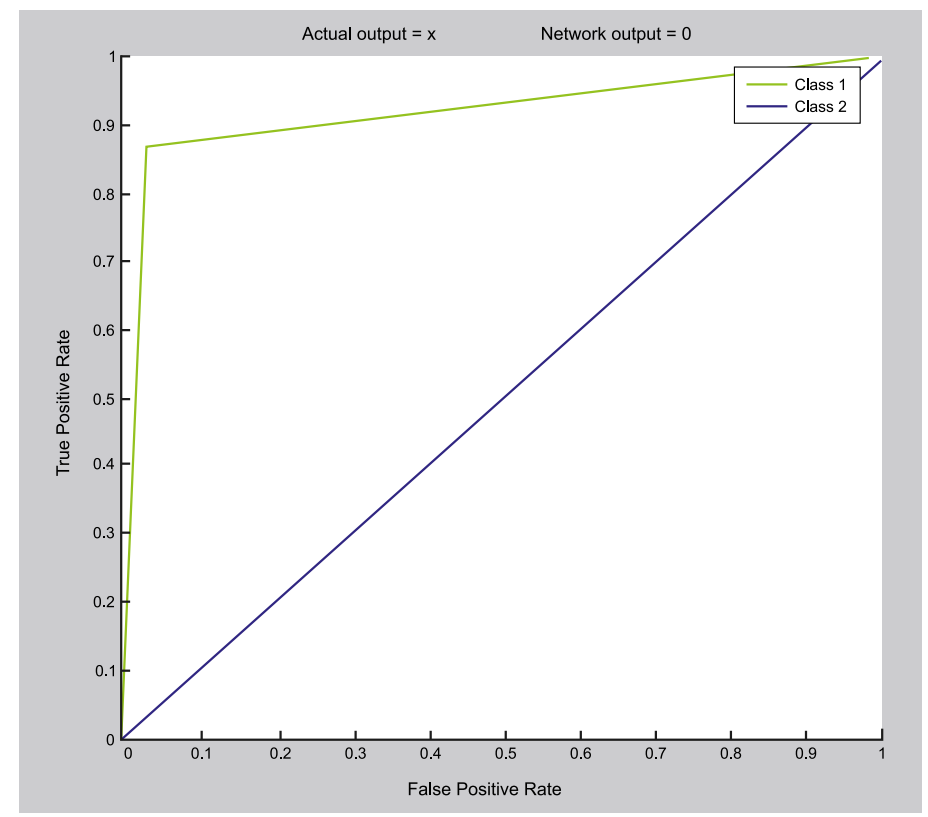

Figure 5. ELM ROC.

\section{ACKNOWLEDGEMENT}

The authors thank the management of Kalasalingam Academy of research and education for granting financial assistance underside the rule of University Research Fellowship (URF) in Department of Electronics and Communication Engineering. Also, we thank KGS health care centre, Madurai for granting the pancreas MR image that is very useful for this research work 


\section{REFERENCES}

Armstrong, T. S., Cohen, M. Z., Weinbrg, J., \& Gilbert, M. R. (2004). Imaging techniques in neuro oncology. Seminars in Oncology Nursing, 20(4), 231-239. https:// doi.org/10.1016/j.soncn.2004.07.003

Aruna Devi, B., \& Pallikonda Rajasekaran, M. (2018). Performance Evaluation of MRI Pancreas Image Classification Using Artificial Neural Network (ANN). In Satapathy S., Bhateja V., Das S. (eds.) Smart Intelligent Computing and Applications. Smart Innovation, Systems and Technologies, vol. 104. Springer, Singapore. https://doi. org/10.1007/978-981-13-1921-1_65

Aruna Devi, B., Pallikonda Rajasekaran, M., \& Thiyagarajan, A. P. (2019). Analysis and classification of malignancy in pancreatic magnetic resonance images using neural network techniques. International journal of imaging systems and technology. https:/ / doi.org/10.1002/ima.22314

Huang, G., Zhu, Q., \& Siew, G. (2004). Extreme learning machine: a new learning scheme of feedforward neural networks. In 2004 IEEE International foint Conference on Neural Networks (IEEE Cat. No.04CH37541), 2, 985-990, vol. 2. https://doi.org/10.1109/ IJCNN.2004.1380068

Ibrahim, W. H., Osman, A. A., \& Mohamed, Y. I. (2013). MRI brain image classification using neural networks. In 2013 International Conference on Computing, Electrical and Electronic Engineering (ICCEEE), 253-258. https://doi.org/10.1109/ ICCEEE.2013.6633943

Kavitha, S., \& Thyagharajan, K. K. (2012). Features based mammogram image classification using weighted feature support vector machine. In Krishna P. V., Babu M. R., Ariwa E. (eds.) Global Trends in Information Systems and Software Applications. ObCom 2011. Communications in Computer and Information Science, vol. 270. Springer, Berlin, Heidelberg. https://doi.org/10.1007/978-3-642-29216-3_35 
Lu, S. S., Qiu, X., Shi, J., Li, N., Lu, Z.H., Chen, P., ... Zhang, Y. D. (2017). A pathological brain detection system based on extreme learning machine optimized by bat algorithm. CNS \& neurological disorders drug targets, 16(1), 23-29. https://doi.org /10.2174/1871527315666161019153259

Nandpuru, H. B., Salankar, S. S., \& Bora, V. R. (2014). MRI brain cancer classification using Support Vector Machine. In 2014 IEEE Students' Conference on Electrical, Electronics and Computer Science, 1-6. https://doi.org/10.1109/SCEECS.2014.6804439

Nazir, M., Wahid, F., \& Khan, S. A. (2015). A simple and intelligent approach for brain MRI classification. Fournal of Intelligent and Fuzzy Systems, 28, 1127-1135. https://doi. org/10.3233/IFS-141396

Othman, M. F., Abdullah, N., \& Kamal, N. F. (2011). MRI brain classification using support vector machine. In 2011 Fourth International Conference on Modeling, Simulation and Applied Optimization, 1-4. https://doi.org/10.1109/ICMSAO.2011.5775605

Shah, J., Surve, S., \& Turkar, V. (2015). Pancreatic Tumor Detection using Image processing Techniques. Procedia Computer Science, 49, 11-16. https://doi.org/10.1016/j. procs.2015.04.221

Vapnik, V. (1995). The Nature of Statistical Learning Theory. New York: Springer.

Yao,J., Chen,J., \& Ghow, G. (2009). Breast Tumor Analysis in Dynamic Contrast Enhanced MRI Using Texture Features and Wavelet Transform. IEEE Journal of Selected Topics in Signal Processing, 3, 94-100. https://doi.org/10.1109/JSTSP.2008.2011110 
